\title{
La transparencia presupuestaria en las comunidades autónomas españolas
}

\author{
Ana-María Ríos \\ Ana-Belén Redondo-López \\ Bernardino Benito
}

RESUMEN: La ciudadanía ha mostrado en los últimos años un interés creciente por conocer que está haciendo el sector público con los recursos que recibe, por lo que las entidades públicas se han visto obligadas a facilitar y poner a su disposición información actualizada sobre la utilización de estos recursos. En este sentido, el propósito del presente trabajo es analizar qué factores socioeconómicos y políticos afectan a los niveles de transparencia, y de forma más concreta la relacionada con los presupuestos de los gobiernos de las diecisiete Comunidades Autónomas españolas para los años 2010, 2012, 2014 y 2016. La elección de los mismos se debe a que son los años para los que Trasparencia Internacional España ha publicado los datos sobre el índice de transparencia económico-financiera de las Comunidades Autónomas.

Los resultados muestran que aquellas Comunidades Autónomas que presentan una mayor población, unas menores tasas de desempleo, un mayor acceso a internet de su población, un saldo presupuestario positivo y que son gobernadas por hombres, tienen unos niveles de transparencia superiores. Sin embargo, los resultados revelan que variables socioeconómicas como el nivel educativo de la población y la deuda de las autonomías no resultan significativas para determinar los niveles de transparencia presupuestaria. De igual manera, variables políticas como la ideología, el grado de participación electoral y la forma de gobierno (mayorías o coaliciones) no son relevantes en el estudio.

PALABRAS CLAVE: Transparencia, presupuestos, gobiernos regionales, comunidades autónomas.

CLAVES ECONLIT: H61, H83.

Cómo citar este artículo / How to cite this article: RíOS, A.M., REDONDO-LÓPEZ, A.M. \& BENITO, B. (2019):

"La transparencia presupuestaria en las comunidades autónomas españolas", CIRIEC-España, Revista de Economía Pública, Social y Cooperativa, 96, 281-310. DOI: 10.7203/CIRIEC-E.96.13835.

Correspondencia: Ana-María Ríos, anamaria.rios1@um.es; Ana-Belén Redondo-López, anabelen.redondo@hotmail.com; Bernardino Benito, benitobl@um.es (autor para correspondencia). Facultad de Economía y Empresa, Universidad de Murcia. 


\section{EXPANDED ABSTRACT}

\section{Budgetary transparency in the spanish autonomous communities}

\section{Objectives}

The main purpose of this research is to analyse the evolution of budgetary transparency in the Spanish Autonomous Communities (ACs) (also known as regions) and determine which socio-economic and political factors favor such evolution. To this aim, we will use the Transparency Index of the Autonomous Communities (INCAU), elaborated by Transparency International Spain, that is based fundamentally on contrasting whether these entities publish information relating to a set of 80 indicators, which try to cover the most important areas of information that the government of an Autonomous Community (AC) should make publicly available to society. In the selection of these 80 indicators, specialists and technicians from numerous institutions have been collaborating (Transparency International Spain, 2018).

Specifically, INCAU has a twofold objective: (i) to evaluate the level of transparency of the governments of the different ACs and (ii) to encourage and promote the increase in the information that these institutions provide to citizens and to society as a whole. This index has been produced every two years since 2010. It only assesses whether the required information is available or not, but it does not evaluate the quality of the information published, nor that of the management of the governments of different ACs. Each AC obtains a score, the INCAU, giving rise to a classification of the level of transparency of the seventeen autonomous governments. INCAU is made up of several transparency sub-indexes: a) Information on the Autonomous Community; b) Relations with citizens and society; c) Economic-financial transparency; d) Transparency in contracts for services, works and supplies; e) Transparency in matters of land use planning, urban planning and public works; f) Indicators of the Transparency Law. In this research we will focus on the area of economic-financial transparency.

Taking into account the previous literature, it has been considered that the socioeconomic factors that can explain the level of transparency are the population, the economic level (unemployment), the educational levels of citizenship, the use of the Internet by citizens, the budget deficit or surplus and the indebtedness. Political factors include the ideology of the ruling political party, the gender of the president, electoral participation and political competition.

\section{Methodology}

In order to measure the degree of budgetary transparency of the Spanish ACs, we use the index of economic-financial transparency published by Transparency International Spain. Therefore, the 
dependent variable of our model is this index, and the data have been obtained for the years 2010, 2012, 2014 and 2016. There are two possible scoring levels in each of the 80 indicators that make up the INCAU: a) 1 point: the information on the indicator is published on the AC website; $b) 0$ points: the information on the indicator is not published on the AC website. Based on the total score obtained by each of the ACs evaluated (the sum of their scores in the 80 indicators), Transparency International Spain prepares the final transparency ranking of the ACs (with a score ranging from 1 to 100, with 100 being the most transparent) both at an overall level and in each of the six areas of transparency that have been evaluated.

For the last year considered (2016), the most transparent AC is the Basque Country, followed by the Balearic Islands, Catalonia and Madrid. On the other hand, on the less transparent side we find ACs such as Navarre, Extremadura or Aragon. In relation to the average of all the years analysed, the ACs with the best data are Catalonia, Castilla-Leon and La Rioja, with the three being above 80 points on average in the assessment of their levels of economic-financial transparency. In addition, those with the lowest levels of transparency on average in the period analysed are Castilla-la Mancha and the Comunidad Valenciana, which scarcely reach a score of 60 points.

In order to analyse the explanatory factors of the levels of budgetary transparency, a linear multi-

ple regression model has been proposed, which is estimated by means of the Ordinary Least Squares (OLS) method.

\section{Results}

The following table shows a summary of the hypotheses initially put forward and whether they have finally been accepted or rejected after comparing them in the model proposed in our methodology. 


\begin{tabular}{|l|l|}
\hline HYPOTHESIS & ACCEPTED/REJECTED \\
\hline $\begin{array}{l}\text { H1: There is a positive relationship between the size of the population of the Autonomous } \\
\text { Communities and the budgetary transparency of their governments. }\end{array}$ & ACCEPTED \\
\hline $\begin{array}{l}\text { H2: There is a relationship between the economic level of the Autonomous Communities } \\
\text { and the budgetary transparency of their governments. }\end{array}$ & ACCEPTED \\
\hline $\begin{array}{l}\text { H3: There is a positive relationship between the educational level of the population of the } \\
\text { Autonomous Communities and the budgetary transparency of their governments. }\end{array}$ & REJECTED \\
\hline $\begin{array}{l}\text { H4: There is a positive relationship between the Internet access of the population of the } \\
\text { Autonomous Communities and the budgetary transparency of their governments. }\end{array}$ & ACCEPTED \\
\hline $\begin{array}{l}\text { H5: There is a relationship between the budgetary balance of the Autonomous Communities } \\
\text { and the budgetary transparency of their governments. }\end{array}$ & ACCEPTED \\
\hline $\begin{array}{l}\text { H6: There is a relationship between the level of indebtedness of the Autonomous } \\
\text { Communities and the budgetary transparency of their governments. }\end{array}$ & REJECTED \\
\hline $\begin{array}{l}\text { H7: There is a relationship between the ideology of the President of the Autonomous } \\
\text { Community and the budgetary transparency. }\end{array}$ & ACCEPTED \\
\hline $\begin{array}{l}\text { H8: There is a relationship between the gender of the President of the Autonomous } \\
\text { Community and the budgetary transparency. }\end{array}$ & REJECTED \\
\hline $\begin{array}{l}\text { H9: There is a positive relationship between citizen participation in regional elections and } \\
\text { budgetary transparency. }\end{array}$ & REJECTED \\
rency. & \\
\hline
\end{tabular}

\section{Conclusions/Limitations/lmplications}

Analysis of the evolution of the ACs' economic-financial transparency index has enabled us to observe that its own publication has been one of the greatest stimuli for governments to increase their level of public information. This is a fact that undoubtedly implies a limitation to the dependent variable used in our model, since the range of variation of this is reduced over time, and many ACs are approaching to the transparency indexes of $100 \%$ in 2016 . Therefore, it is possible that, over time and with the publication of new transparency indexes, the model proposed in this study will no longer be significant to the extent that the dependent variable (the transparency index) will tend to be uniform (the value of the index of all the ACs will be or will become 100\%). In addition, it should not be forgotten that another of the limitations of these indicators is that the quality of the information issued by governments is not included in their analysis, but only the publication of this information itself. 
It should also be noted that any dissemination involves several costs, mainly in terms of staff time dedicated to the preparation of the information. However, much of the information is prepared periodically for compliance with obligations to other administrative bodies. That is why we believe that the advance of transparency in the field of autonomy is a question of predisposition, will and know-how, rather than of significant technological investments.

In this sense, we also believe that regional governments should opt to continue improving their levels of transparency, especially those ACs of smaller size, as we have concluded in the study that they are the ones with lower levels of transparency. This can be achieved through improvements in the means by which they communicate information. Thus, as we have concluded, the population's access to the Internet is a factor that very positively affects the levels of transparency. Therefore, ACs must put emphasis on this type of means in order to provide quality information with sufficient clarity to the citizens.

Normally, institutions tend to make data public, but not understandable to a large part of the citizenry. For this reason, the role of citizens is to continue asking for exhaustive, clear and complete information about the management of public administrations.

As future areas of research that allow us to complement and deepen the study of the levels of transparency, the creation of new indexes would be positive in order to check the similarities and differences between them, including in the calculation of these indexes the quality and clarity of the information published by the public administrations. It would also be positive to analyse the evolution of the transparency index through panel data when there is a longer period of time.

KEYWORDS: Transparency, budgets, regional governments, Autonomous Communities. 


\section{Introducción}

En los últimos años se ha generalizado en la sociedad española una sensación de desconfianza hacia los gobiernos de las entidades públicas debido fundamentalmente a la crisis económica y a los innumerables casos de corrupción que han aparecido.

Lo anterior ha provocado un interés creciente en la ciudadanía por conocer en qué se están gastando los recursos que se aportan a estas organizaciones, las cuales se han visto obligadas a facilitar información actualizada sobre la utilización de los mismos. De hecho, en las últimas décadas, la reforma de los sistemas de gestión pública se ha centrado en reforzar la responsabilidad de las instituciones públicas, siendo la transparencia un requisito fundamental (Kopits y Craig, 1998).

El presupuesto es el instrumento de política económica más importante de los gobiernos de las entidades públicas, dado que refleja las prioridades de las políticas económicas y sociales más que ningún otro instrumento, traduciendo políticas, compromisos políticos y metas en decisiones sobre en qué se deben gastar los recursos obtenidos. El presupuesto de las economías modernas es muy complejo, a veces innecesariamente, lo que permite a los políticos implementar prácticas que intentan "ocultar" el equilibrio presupuestario real (Alesina y Perotti, 1996). De hecho, los políticos tienen incentivos para ocultar los impuestos, enfatizar los beneficios del gasto público y encubrir las obligaciones futuras del gobierno. Sin embargo, los estímulos para realizar el presupuesto de forma clara y transparente son bastante escasos.

Por tanto, se requiere de mecanismos de rendición de cuentas y sistemas de información que reflejen de la manera más integral posible la forma en la que el gobierno cumple con sus responsabilidades. En este sentido, la transparencia presupuestaria, según el Fondo Monetario Internacional (FMI) (2012), se puede definir como la claridad, fiabilidad, frecuencia, oportunidad y relevancia de los informes fiscales públicos y la apertura a la ciudadanía del proceso de formulación de las políticas fiscales del gobierno, siendo un elemento fundamental de la gestión fiscal eficiente.

Según Mitchell (1998), existen numerosos trabajos que estudian la transparencia de los gobiernos en el ámbito internacional, si bien no ha ocurrido lo mismo para el caso concreto de la transparencia presupuestaria. Algunos trabajos como el de Ríos et al. (2014) analizan el papel que desempeña la supervisión presupuestaria, así como otros factores socioeconómicos y políticos, en la mejora de la transparencia presupuestaria a nivel internacional. En el ámbito local, también existen algunos trabajos como el de Pallot (2001), Pina et al. (2007), o Guillamón et al. (2011), que analizan los niveles de transparencia presupuestaria con la finalidad de arrojar mayor información sobre sus determinantes en las administraciones locales. Para el caso de España, en concreto, existen diversos estudios 
sobre transparencia presupuestaria a nivel local (Guillamón et al., 2011; Polo-Otero, 2011; EstellerMoré y Polo-Otero, 2012; Navarro et al., 2016), pero hasta lo que nosotros sabemos no hay ninguno que analice sus determinantes a nivel autonómico, si bien es cierto que el trabajo de Bellod (2008) pretende evaluar el grado de transparencia presupuestaria de las Comunidades Autónomas (CCAA) españolas.

El propósito de este trabajo es, por tanto, analizar la evolución de la transparencia presupuestaria de las CCAA españolas y conocer qué factores socioeconómicos y políticos favorecen la misma. Para ello, utilizaremos el Índice de Transparencia de las Comunidades Autónomas (INCAU), elaborado por Transparencia Internacional España, que se basa fundamentalmente en contrastar si estas instituciones publican la información relativa a un conjunto de 80 indicadores, que tratan de abarcar las áreas más importantes de la información que los responsables del gobierno de una Comunidad Autónoma (CA) deberían poner públicamente a disposición de la sociedad. En la selección de estos 80 indicadores han venido colaborando especialistas y técnicos de numerosas instituciones (Transparencia Internacional España, 2018).

En concreto, el INCAU tiene un doble objetivo: (i) realizar una evaluación del nivel de transparencia de los Gobiernos de las diferentes CCAA y (ii) propiciar e impulsar el aumento de la información que estas instituciones ofrecen a los ciudadanos y a la sociedad en su conjunto. Este índice se elabora con periodicidad bienal desde el año 2010. Sólo se valora si la información requerida está 0 no disponible, pero no evalúa la calidad de la información publicada, ni la de la gestión de los gobiernos de las CCAA. Cada CA obtiene una puntuación, el INCAU, dando origen a una clasificación del nivel de transparencia de los diecisiete gobiernos autonómicos. El INCAU está formado a su vez por varios subíndices de transparencia: a) Información sobre la Comunidad Autónoma; b) Relaciones con los ciudadanos y la sociedad; c) Transparencia económico-financiera; d) Transparencia en las contrataciones de servicios, obras y suministros; e) Transparencia en materias de Ordenación del territorio, urbanismo y obras públicas; f) Indicadores del Proyecto de Ley de Transparencia (estatal). En la presente investigación nos vamos a centrar en el área de transparencia económico-financiera, que es la que nos interesa.

La estructura de este trabajo es la que sigue. Tras esta introducción, el estudio contiene un apartado de literatura previa, donde se presenta la investigación sobre los determinantes de la transparencia, tanto socioeconómicos como políticos. El apartado de metodología contiene la muestra, las variables y el modelo. A continuación, se exponen los resultados del estudio. Finalmente, se indican las principales conclusiones e implicaciones del mismo. 


\section{Literatura previa}

Según la literatura previa, existen diferentes factores que permiten explicar los niveles de transparencia de los gobiernos, si bien, y hasta donde nosotros conocemos, en el caso de las CCAA apenas existen trabajos al respecto, por lo que a la hora de establecer las variables que pueden explicar el nivel de transparencia en estas administraciones tendremos que extrapolar lo que se ha dicho al respecto en los gobiernos centrales y locales. Así, agruparemos estas variables o factores explicativos en dos grandes grupos: socioeconómicos y políticos.

\subsection{Factores socioeconómicos}

Entre los factores socioeconómicos que afectan a los niveles de transparencia nos encontramos la población, el nivel económico, los niveles educativos de la ciudadanía, el uso de internet por parte de la ciudadanía, el déficit o superávit presupuestario y el endeudamiento.

Diversos estudios han considerado el tamaño poblacional como un factor explicativo de los niveles de transparencia (Evans y Patton, 1987; Ingram y DeJond, 1987). Según la Teoría de la Agencia, las asimetrías de información entre políticos y ciudadanos suelen ser más elevadas en municipios de gran tamaño. Por tanto, estos gobiernos tienen incentivos para divulgar una mayor cantidad de información con el fin de reducir estas asimetrías y permitir a los ciudadanos evaluar su actuación (Zimmerman, 1977). Por su parte, la Teoría Institucional postula que los gobiernos de municipios más grandes suelen estar más presionados para que divulguen información (Ríos et al., 2013). Además, en municipios con una mayor población existe más demanda de servicios públicos que en los menos habitados, por lo que los gobernantes estarán más interesados en divulgar información pública y así conseguir una mayor transparencia en la gestión de estos servicios (Guillamón et al., 2011).

Trabajos como el de Guillamón et al. (2011) y García-Tabuyo et al. (2015) encuentran una relación significativa y positiva entre el tamaño de la población y los niveles de transparencia. Por su parte, Ingram y DeJong (1987) no han conseguido demostrar que exista una relación estadísticamente significativa entre el tamaño poblacional y la divulgación de información. Dicho lo anterior, y extrapolando lo que postulan los estudios previos para los gobiernos locales y centrales, presentamos la siguiente hipótesis para el caso de las autonomías:

H1: Existe una relación positiva entre el tamaño de la población de las CCAA y la transparencia presupuestaria de sus gobiernos. 
En cuanto al nivel económico, algunos autores como Grigorescu (2003) afirman que en los países más desarrollados no es frecuente la preocupación por el elevado coste que puede suponer la recopilación, procesamiento y divulgación de información, por lo que son más proclives a adoptar leyes sobre revelación de información, lo que les hace más transparentes. Por su parte, Christiaens (1999) argumenta que dado que los ciudadanos consideran que aquellos municipios que presentan mayor riqueza económica son los que están mejor gestionados, se espera que propaguen mayor cantidad de información. En este mismo sentido, Giroux y McLelland (2003) afirman que los ciudadanos con mayor poder adquisitivo tienden a prestar mayor atención a la gestión que realizan los políticos, lo que les lleva a demandar más información sobre cómo administran los recursos. Sin embargo, Grigorescu (2003) sostiene por otro lado que los ciudadanos de los países pobres tienen más incentivos para solicitar información, ya que su grado de satisfacción con la actuación del gobierno es menor.

Estudios empíricos previos como el de Huther y Shah (1998) y el de Arikan (2004), muestran que cuanto más rico es un país, más transparente es su presupuesto. En el mismo sentido, otros autores han demostrado que el nivel económico está relacionado positivamente con la transparencia fiscal (Hammed, 2005; Piotrwski y Van Ryzin 2007). Sin embargo, Alt et al. (2006) no encuentran una relación significativa entre estas variables. Por tanto, a la vista de lo anterior, planteamos la siguiente hipótesis para el caso de las CCAA:

\section{H2: Existe una relación entre el nivel económico de las CCAA y la transparencia presu- puestaria de sus gobiernos.}

Respecto al nivel de educación de la población, Tolbert y Mossberger (2006) señalan que una población con alto nivel educativo demandará mayor volumen de información a las administraciones públicas. En esta misma línea, Junquera (2013) establece que es necesario educar a la población desde la escuela en conceptos de honestidad que incluyan un modelo de honradez fiscal. De esta manera, las poblaciones mejor educadas exigirán a sus gobernantes más transparencia en sus acciones.

Desde el punto de vista empírico, Gandía y Archidona (2008) y Navarro et al. (2016) identifican el nivel educativo que poseen los ciudadanos como uno de los factores positivos y decisivos para la publicación de información en la web de los municipios. Sin embargo, tanto Ma y Wu (2011), en su estudio relativo a las provincias chinas, como Serrano et al. (2009), no encuentran relación significativa entre el nivel educativo y la publicación de información. A la vista de lo que dice la mayor parte de la literatura, se plantea la siguiente hipótesis para el caso de las CCAA:

\footnotetext{
H3: Existe una relación positiva entre el nivel educativo de la población de las CCAA y la transparencia presupuestaria de sus gobiernos.
} 
Otro determinante interesante para el análisis de los niveles de transparencia es el uso de internet. Según postula Kim (2007), cuando el número de ciudadanos con acceso a internet es elevado, la posibilidad de que éstos visiten las webs de sus gobiernos y, por consiguiente, demanden una mayor información a través de ellas, es mayor.

Estudios empíricos como el de García (2014) confirman que la tecnología y la cultura digital se están instalando en la sociedad, creando unas instituciones más transparentes y con procesos más democráticos, y prestando especial atención al concepto de datos públicos abiertos como base de la estrategia de apertura gubernamental y gestión pública. Así, para el caso de las CCAA, de acuerdo con la literatura previa, se plantea la siguiente hipótesis:

\section{H4: Existe una relación positiva entre el acceso a internet de la población de las CCAA y la transparencia presupuestaria de sus gobiernos.}

Por su parte, a pesar del potencial del resultado presupuestario para valorar la calidad de la gestión, esta variable no ha sido incluida de manera habitual en la literatura sobre transparencia. No obstante, es fácil suponer que cuanto más deficiente sea la gestión presupuestaria, medida, por ejemplo, por este resultado presupuestario, menores serán los incentivos y recursos para sostener una política de transparencia, aunque también es cierto que ésta podría ser utilizada para ganar una legitimidad que actuara de pantalla frente a esa mala gestión (Alonso y García, 2014). En este sentido, Ma y Wu (2011) encuentran una relación positiva altamente significativa entre el déficit presupuestario y la transparencia, es decir, las provincias chinas con mayor déficit son las que más información facilitan. Benito y Bastida (2009) también encuentran una relación positiva y significativa entre el resultado presupuestario y la transparencia presupuestaria. En nuestro país, tanto Esteller-Moré y Polo-Otero (2012) como Albalate (2013) no hallan un impacto estadísticamente significativo del déficit fiscal en la transparencia presupuestaria de los gobiernos locales. Por tanto, dado que la literatura no es clara en cuanto a la relación que existe entre saldo presupuestario y transparencia, se plantea la siguiente hipótesis para el caso de las CCAA:

\section{H5: Existe una relación entre el saldo presupuestario de las CCAA y la transparencia pre- supuestaria de sus gobiernos.}

Finalmente, el nivel de endeudamiento se ha considerado tradicionalmente como un factor explicativo de los niveles de transparencia de la información. Según la Teoría de Agencia, los políticos tienen incentivos para reducir el coste de la deuda con el fin de incrementar los recursos disponibles para otros programas que mejoren su propio bienestar, por lo que se espera que éstos divulguen más información para disminuir las asimetrías que pueden surgir entre ellos y los acreedores, consiguiendo así reducir el riesgo percibido por estos últimos y disminuir el coste de los recursos ajenos (Zimmerman, 1977). Por otra parte, cuando la deuda alcanza un nivel insostenible y surgen las primeras dificulta- 
des financieras, publicar informes que reflejen la situación financiera de los organismos públicos adquiere una mayor importancia (Wilson y Howard, 1985).

Desde el punto de vista empírico, los resultados de los estudios existentes no son concluyentes. Mientras que los trabajos de Evans y Patton (1987) o Ingram y DeJong (1987) muestran que existe una relación positiva entre la deuda y la divulgación de información, Alt et al. (2006) indican que el nivel de deuda está negativamente relacionado con la transparencia fiscal. Por su parte, Guillamón et al. (2011) no encuentran una relación estadísticamente significativa entre el nivel de endeudamiento y la transparencia presupuestaria de los gobiernos locales. Por tanto, a la vista de lo anterior, plateamos la siguiente hipótesis:

H6: Existe una relación entre el nivel de endeudamiento de las CCAA y la transparencia presupuestaria de sus gobiernos.

\subsection{Factores políticos}

Otro grupo de determinantes de los niveles de transparencia son los relacionados con los factores políticos, destacando en la literatura la ideología del partido de gobierno, el género del presidente, la participación electoral y la competencia política.

La ideología del partido político gobernante ha sido considerada como un determinante clásico de la transparencia. Así, Ferejohn (1999) argumenta que aquellos políticos que deseen aumentar el tamaño del sector público deben incrementar la transparencia con el fin de que los votantes les confíen una mayor cantidad de recursos. Por tanto, dado que los gobiernos progresistas abogan por un sector público amplio y convenientemente financiado, estarán dispuestos a implementar mayores niveles de transparencia que los conservadores.

Desde el punto de vista empírico, Guillamón et al. (2011) encuentran que los partidos de izquierdas son más transparentes que los de derechas. En el mismo sentido, Albalate (2013) halla una relación positiva entre los gobiernos progresistas y un mayor suministro de información. Por su parte, Bastida y Benito (2007), Serrano et al. (2009) y Navarro et al. (2016) muestran que no existen diferencias en el nivel de transparencia como consecuencia de la ideología. En este sentido, para el caso de los gobiernos autonómicos planteamos la siguiente hipótesis:

\section{H7: Existe una relación entre la ideología del presidente de la CA y la transparencia presu- puestaria}


La literatura previa también ha analizado la relación entre el género de los que ostentan cargos políticos de relevancia y los niveles de transparencia. Autores como Verba (1978), sostienen que los hombres suelen estar más involucrados en la actividad política que las mujeres. Sin embargo, cabe señalar que este tipo de análisis se encuentra desfasado en la actualidad, debido al creciente y necesario peso de la mujer en cargos públicos de relevancia. Así, desde perspectivas relativamente más actuales como la de La Porte et al. (2002), se afirma que las culturas machistas tienden a solucionar los problemas de forma colectiva, mientras que las feministas se orientan a resolverlos mediante el compromiso y la negociación. Por tanto, se espera que los países más avanzados en igualdad de género sean más propensos a proporcionar más información a la ciudadanía, dado que en éstos priman valores como compartir o entablar relaciones entre los distintos bloques sociales. Por tanto, siguiendo a la literatura previa, se plantea la siguiente hipótesis:

\section{H8: Existe una relación entre el género del presidente de la CA y la transparencia presu- puestaria.}

Por otra parte, uno de los factores políticos que puede afectar a los incentivos de los gobernantes para divulgar información es el grado de participación de los ciudadanos en los procesos electorales, teniendo en cuenta el interés de éstos por las actividades que realizan los políticos, por lo que un incremento de la trasparencia será bien visto por los votantes (Serrano-Cinca et al., 2009).

Desde un punto de vista empírico, Polo-Otero (2011) encuentra una significatividad positiva entre estas dos variables. A la vista de lo anterior, planteamos la siguiente hipótesis para el caso de las CCAA:

\section{H9: Existe una relación positiva entre la participación de los ciudadanos en las eleccio- nes autonómicas y la transparencia presupuestaria.}

Finalmente, otro factor político muy estudiado en la literatura sobre transparencia es la competencia política. Se entiende que los partidos políticos que ganan las elecciones por mayorías absolutas, es decir, gobiernos en solitario sin necesidad de acuerdos ni coaliciones, tendrán unos niveles de transparencia mucho más bajos que los partidos que para gobernar tienen que llegar a acuerdos (Guillamón et al., 2011).

Existen diferentes trabajos empíricos que estudian la relación entre la competencia política y la transparencia, encontrándose conclusiones muy dispares entre ellos. Así, Serrano et al. (2009) encuentran una significatividad positiva entre una mayor competencia electoral y la rendición de cuentas por parte de gobiernos y administraciones. Por su parte, Guillamón et al. (2011) hallan igualmente que la fragmentación del gobierno está relacionada con una mayor transparencia. Sin embargo, Gallego 
et al. (2010) y Cuadrado (2014), no encuentran relación significativa entre competencia política y transparencia. Por tanto, a la vista de lo anterior, plateamos la siguiente hipótesis para el caso de las CCAA:

H10: Existe una relación entre el mayor grado de competencia política y la transparencia presupuestaria.

\section{Metodología}

\subsection{Muestra}

Tal y como se ha dicho anteriormente, el objetivo de este trabajo es analizar qué factores socioeconómicos y políticos afectan al nivel de transparencia presupuestaria de las CCAA españolas.

Los datos han sido obtenidos para los años 2010, 2012, 2014 y 2016, ya que son los únicos para los que la organización Trasparencia Internacional España publica el índice sobre transparencia económico-financiera de los gobiernos autonómicos.

Los datos de las variables socioeconómicas proceden de diversas fuentes. Por un lado, la población, el nivel económico de las CCAA, el nivel educativo y el uso de internet se han extraído de la base de datos del Instituto Nacional de Estadística (INE). Por otra parte, los datos referentes al déficit o superávit presupuestario y al nivel de endeudamiento han sido obtenidos de los informes del Ministerio de Hacienda.

Los datos de las variables políticas, como la ideología del partido de gobierno, el género del presidente autonómico, la participación electoral y si se gobierna en mayoría o en coalición, han sido extraídos de los informes que emite el Ministerio del Interior.

\subsection{Variables}

\subsubsection{Variable dependiente}

Para medir el grado de transparencia presupuestaria de las CCAA españolas vamos a utilizar el índice de transparencia económico-financiera publicado por Transparencia Internacional España. Por tanto, la variable dependiente de nuestro modelo será este índice (trans_pres). 
El índice de transparencia económico-financiera, tal y como ya hemos comentado en la introducción de este trabajo, es uno de los subíndices que componen el Índice de Transparencia de las Comunidades Autónomas (INCAU). A través del mismo se busca propiciar un mayor acercamiento de las instituciones autonómicas a la sociedad española, fomentando el aumento de la información que los ciudadanos reciben de las mismas en relación con el gobierno y la administración de las CCAA (Transparencia Internacional España, 2018).

El procedimiento de elaboración de este índice se basa en contrastar si estas instituciones publican la información relativa a un conjunto de 80 indicadores, que tratan de abarcar las áreas más importantes de la información que los responsables del gobierno de una CA deberían poner públicamente a disposición de la sociedad. Hay dos posibles niveles de puntuación en cada uno de los 80 indicadores que integran el INCAU: a) 1 punto: la información del indicador está publicada en la web de la $\mathrm{CA}$; b) 0 puntos: la información del indicador no se publica en la web de la CA. En función de la valoración total obtenida por cada una de las CCAA evaluadas (la suma de su puntuación en los 80 indicadores), Transparencia Internacional España elabora la clasificación o ranking final de transparencia de dichas CCAA (con una puntuación que va de 1 a 100, siendo 100 lo más transparente) tanto a nivel global, como en cada una de las seis áreas de transparencia que se han evaluado. Así, a partir de la situación global de cada gobierno autonómico, con el INCAU se llegan a conocer, además, aquellos aspectos en los cuales los organismos a nivel colectivo presentan mayores o menores niveles de transparencia en cuanto a su información económico-financiera, contratación de servicios y suministros, ordenación del territorio, etc. (Transparencia Internacional España, 2018). En este estudio, tal y como hemos dicho, vamos a utilizar solamente el índice de transparencia económico-financiera.

La tabla 1 presenta el índice de transparencia económico-financiera de las diecisiete CCAA para los cuatro años en los que está disponible.

Para el último año considerado (2016), la CA más transparente es el País Vasco, seguida de Baleares, Cataluña y Madrid. Por su parte, en el lado de las menos transparentes encontramos CCAA como Navarra, Extremadura o Aragón. En relación a la media de todos los años analizados, aquellas CCAA que presentan un mejor dato son Cataluña, Castilla y León y La Rioja, situándose las tres por encima de 80 puntos de media en la valoración de sus niveles de transparencia económico-financiera. Por otro lado, aquellas que menores niveles de transparencia presentan de media en el periodo analizado son Castilla la Mancha y la Comunidad Valenciana, que apenas alcanzan una valoración de 60 puntos. 


\section{Tabla 1. Evolución niveles de transparencia económico- financiera}

\begin{tabular}{|c|c|c|c|c|c|c|}
\hline \multirow[t]{2}{*}{ CCAA } & \multicolumn{4}{|c|}{ trans_pres } & \multirow[t]{2}{*}{ MEDIA } & \multirow{2}{*}{$\begin{array}{c}\text { Tasa variación } \\
\text { en } \%(4) /(1)\end{array}$} \\
\hline & 2010 (1) & 2012 (2) & 2014 (3) & 2016 (4) & & \\
\hline Andalucía & 82,40 & 78,60 & 53,80 & 93,80 & 77,15 & 13,83 \\
\hline Aragón & 41,20 & 50,00 & 69,20 & 87,50 & 61,98 & 112,38 \\
\hline Asturias & 52,90 & 42,90 & 76,90 & 96,90 & 67,40 & 83,18 \\
\hline Islas Baleares & 41,20 & 50,00 & 84,60 & 100,00 & 68,95 & 142,72 \\
\hline Canarias & 29,40 & 42,90 & 76,90 & 96,90 & 61,53 & 229,59 \\
\hline Cantabria & 23,50 & 85,70 & 61,50 & 100,00 & 67,68 & 325,53 \\
\hline Castilla y León & 41,20 & 100,00 & 100,00 & 90,60 & 82,95 & 119,90 \\
\hline Castilla-La Mancha & 35,30 & 35,70 & 61,50 & 90,60 & 55,78 & 156,66 \\
\hline Cataluña & 58,80 & 78,60 & 100,00 & 96,90 & 83,58 & 64,80 \\
\hline C. Valenciana & 29,40 & 35,70 & 92,30 & 84,40 & 60,45 & 187,07 \\
\hline Extremadura & 52,90 & 92,90 & 46,20 & 87,50 & 69,88 & 65,41 \\
\hline Galicia & 64,70 & 71,40 & 84,60 & 100,00 & 80,18 & 54,56 \\
\hline C. de Madrid & 70,60 & 78,60 & 61,50 & 100,00 & 77,68 & 41,64 \\
\hline Región de Murcia & 82,40 & 42,90 & 53,80 & 100,00 & 69,78 & 21,36 \\
\hline C. de Navarra & 58,80 & 100,00 & 84,60 & 75,00 & 79,60 & 27,55 \\
\hline País Vasco & 17,60 & 100,00 & 100,00 & 100,00 & 79,40 & 468,18 \\
\hline La Rioja & 47,10 & 100,00 & 84,60 & 100,00 & 82,93 & 112,31 \\
\hline
\end{tabular}

Si analizamos la evolución en el periodo que ocupa nuestro estudio, se observa que todas las CCAA han experimentado un aumento en el índice de transparencia económico-financiera. De hecho, la mayor parte de CCAA supera el $100 \%$ respecto a sus niveles de inicio. Aquellas que muestran mayores crecimientos en estos años son País Vasco (468,18\%), Cantabria (325,53\%) y Canarias $(229,59 \%)$. También ha mejorado el nivel de transparencia presupuestaria de comunidades como Castilla-La Mancha o Comunidad Valenciana, ambas por encima del $150 \%$. Por su parte, en el caso de comunidades como Andalucía y Murcia su crecimiento ha sido menor, ya que partían de unos niveles de transparencia superiores al resto de CCAA. Sin embargo, Navarra, Asturias, Cataluña o Extremadura, pese a tener inicialmente unos niveles relativamente bajos de transparencia, han experimentado un crecimiento bajo. Una posible explicación a esta evolución positiva del índice de transparencia económico-financiero de las CCAA es que la propia publicación de éste ha sido uno de los mayores estí- 
mulos para que los gobiernos incrementen su nivel de información pública. Es decir, la propia existencia de un índice público de transparencia orienta la actuación de los gobiernos autonómicos con el propósito de conseguir una mayor puntuación.

En media, en el periodo 2010-2016, los niveles de transparencia autonómica han crecido en torno a un $12 \%$ anual, un aumento considerable entendido en un contexto en donde existe un interés creciente por parte de la ciudadanía por conocer que está haciendo el sector público con los recursos que recibe.

\subsubsection{Variables independientes}

Las variables independientes que se especifican a continuación han sido seleccionadas a partir de la literatura previa estudiada y teniendo en cuenta las fuentes estadísticas disponibles. De acuerdo con esta literatura, los factores que influyen en la transparencia presupuestaria los hemos dividido en dos categorías: socioeconómicos y políticos.

En cuanto a los primeros, se ha venido considerando de manera tradicional al tamaño de la población como una variable significativa en el estudio de los niveles de transparencia (Evans y Patton, 1987; Ingram y DeJond, 1987; Sáez-Martín et al., 2017). Por este motivo, hemos incluido el tamaño poblacional (población) como variable independiente, y esperamos que esté positivamente relacionado con los niveles de transparencia presupuestaria.

Para medir el nivel económico de las CCAA hemos utilizado la variable desempleo, calculada como el tanto por ciento de población activa que no encuentra empleo. De acuerdo con la literatura previa, el nivel económico también puede ser medido a través de otras variables, entre las que ocupa un lugar destacado el PIB per cápita (en nuestro trabajo la denominamos nivel_eco). No obstante, como veremos en la tabla 3 , se ha descartado esta opción porque está muy correlacionada con otras variables independientes y el modelo podría sufrir de multicolinealidad, por lo que finalmente hemos considerado más oportuno medir el nivel económico con una proxy como es el desempleo, muy utilizado también en estudios anteriores. La literatura previa no ha sido concluyente en cuanto a cómo afecta el nivel económico a los niveles de transparencia presupuestaria, por lo que el signo esperado para esta variable no está claramente definido.

El nivel educativo también ha sido incluido en el modelo mediante la variable nivel_edu. Ésta mide el tanto por ciento de la población con estudios superiores. Se espera que exista una relación positiva entre esta variable y los niveles de transparencia presupuestaria de las CCAA.

Otra variable considerada en el modelo es la denominada internet, con la que se pretende tener en cuenta el grado de uso de internet por parte de los ciudadanos. En concreto, esta variable mide 
el tanto por ciento del total de población que tiene al menos un ordenador, esperando que se relacione positivamente con los niveles de transparencia presupuestaria.

Dentro de las variables socioeconómicas hemos incluido finalmente dos que muestran la situación económico-financiera de los gobiernos autonómicos: la capacidad o necesidad de financiación de las autonomías (saldo_pres_pc) y el nivel de endeudamiento de éstas (deuda_pc), ambas medidas en términos per cápita. El efecto de estas dos variables sobre los niveles de transparencia presupuestaria no ha sido determinado con claridad en la literatura previa. Por tanto, no podemos prever de forma clara el signo de las mismas.

Respecto a los factores políticos que explican la transparencia presupuestaria, la variable ideología es una variable binaria que toma el valor 1 si el partido de gobierno es progresista y 0 cuando es conservador. El signo esperado de esta variable es incierto, ya que su efecto sobre los niveles de transparencia no ha sido determinado de manera unánime por la literatura.

El género del presidente ha sido incluido en el modelo mediante la variable binaria gen_presidente, tomando el valor 1 cuando el género del presidente es femenino y el valor 0 si es masculino. La influencia del género de los cargos públicos sobre los niveles de transparencia presupuestarios tampoco ha resultado significativa en la literatura, por lo que se desconoce a priori cual será el signo de esta variable.

La participación electoral (part_electoral) ha sido definida como el tanto por ciento de población con derecho a votar que ha ejercido el mismo en las correspondientes elecciones. Esperamos que esta participación tenga un impacto positivo en los niveles de transparencia presupuestaria autonómicos.

Por último, para tener en cuenta la competencia política en los niveles de transparencia presupuestaria hemos incluido la variable binaria mayoría. Ésta toma el valor 1 cuando el gobierno gobierna con mayoría y 0 en otro caso. Según la literatura previa, esperamos que cuanto mayor sea la competencia política, mayores sean los niveles de transparencia presupuestaria.

La tabla 2 muestra un resumen de todas las variables utilizadas, los estadísticos descriptivos de éstas, así como el signo esperado de acuerdo con la literatura existente. 


\begin{tabular}{|c|c|c|c|c|c|c|c|c|c|c|c|c|}
\hline 离 & & $\Phi$ & 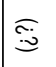 & $\underset{\sim}{\stackrel{\approx}{*}}$ & 王 & \pm & $\underset{\widetilde{\sigma}}{\tilde{\Xi}}$ & 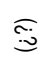 & $\widetilde{\approx}$ & 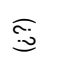 & $\Xi$ & $I$ \\
\hline 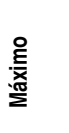 & $\begin{array}{l}8 \\
8 \\
\end{array}$ & $\begin{array}{l}8 \\
0 \\
\mathscr{0} \\
0 \\
0 \\
\\
\infty \\
\infty\end{array}$ & 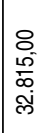 & $\begin{array}{l}\infty \\
\stackrel{\infty}{f} \\
\text { f }\end{array}$ & $\begin{array}{l}\text { 员 } \\
\text { o్ల }\end{array}$ & $\begin{array}{l}\stackrel{0}{1} \\
\stackrel{\infty}{\infty}\end{array}$ & 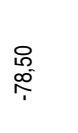 & $\begin{array}{l}\text { g } \\
\infty \\
\infty \\
\infty \\
\infty \\
\infty\end{array}$ & 음 & 음 & $\begin{array}{l}8 \\
\text { O. } \\
\text { O. }\end{array}$ & $\underset{-}{8}$ \\
\hline 茞 & $\begin{array}{l}\stackrel{8}{\circ} \\
\stackrel{-}{\circ}\end{array}$ & 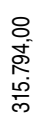 & 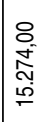 & $\begin{array}{l}\text { \& } \\
\text { : }\end{array}$ & $\stackrel{\stackrel{\circ}{\circ}}{\stackrel{2}{\circ}}$ & $\begin{array}{l}8 \\
8 \\
\& \\
8\end{array}$ & 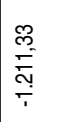 & 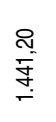 & 8 & 응 & $\frac{10}{5}$ & 8 \\
\hline 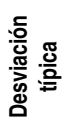 & $\begin{array}{l}\stackrel{2}{\sim} \\
\stackrel{\sim}{\sim}\end{array}$ & 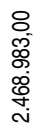 & 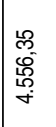 & 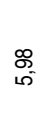 & $\stackrel{\widehat{m}}{\tilde{\omega}^{\circ}}$ & $\frac{\cong}{\circ}$ & $\begin{array}{l}\infty \\
\stackrel{\infty}{N} \\
\stackrel{\sim}{N}\end{array}$ & $\begin{array}{l}\stackrel{\mathscr{D}}{\infty} \\
\text { ஸ్ } \\
\stackrel{\leftrightarrow}{-}\end{array}$ & 守 & 心్ & $\begin{array}{l}\hat{\infty} \\
\text { os } \\
\omega^{\circ}\end{array}$ & $\begin{array}{l}0 \\
0 \\
0 \\
0\end{array}$ \\
\hline $\begin{array}{l}\frac{\pi}{\bar{\sigma}} \\
\frac{\mathrm{d}}{2}\end{array}$ & 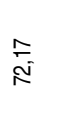 & $\begin{array}{l}\frac{8}{m} \\
\frac{m}{m} \\
\stackrel{8}{+} \\
\stackrel{N}{j}\end{array}$ & 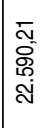 & $\begin{array}{l}\hat{N} \\
\text { 命 }\end{array}$ & $\begin{array}{l}\bar{\sigma} \\
\text { مू }\end{array}$ & $\begin{array}{l}\text { fo } \\
\substack{0 \\
0}\end{array}$ & $\frac{\mathfrak{N}}{\stackrel{\sim}{y}}$ & $\begin{array}{l}\text { f } \\
\text { 志 } \\
\text { o }\end{array}$ & ల్ల & $\frac{0}{0}$ & $\begin{array}{l}8 \\
8 \\
0 \\
\end{array}$ & 胥 \\
\hline 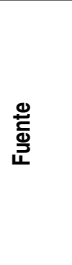 & 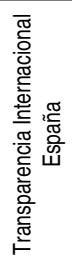 & 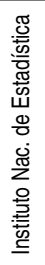 & 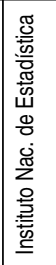 & 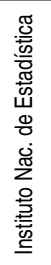 & 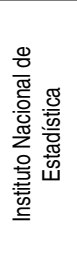 & 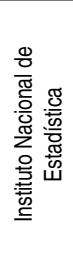 & 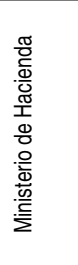 & 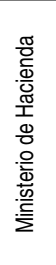 & 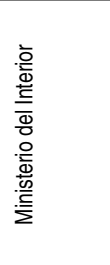 & 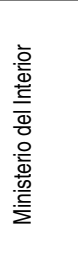 & 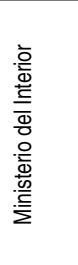 & 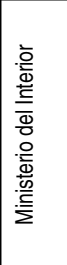 \\
\hline 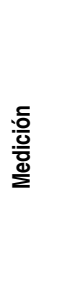 & 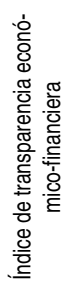 & 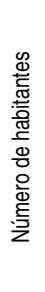 & 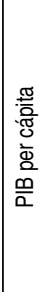 & 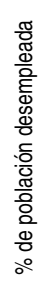 & 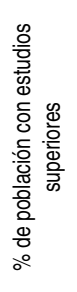 & 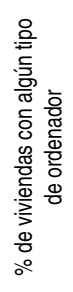 & 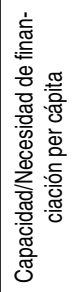 & 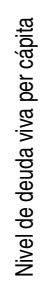 & 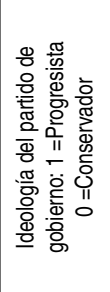 & 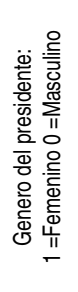 & 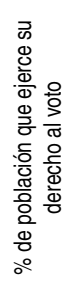 & 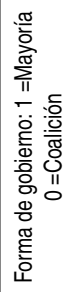 \\
\hline \multirow{2}{*}{$\begin{array}{l}\frac{0}{0} \\
\frac{0 \pi}{2} \\
\frac{\pi}{7}\end{array}$} & 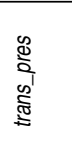 & $\begin{array}{l}\frac{\overline{0}}{\frac{\pi}{2}} \\
\frac{\pi}{2} \\
\frac{8}{2}\end{array}$ & 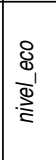 & 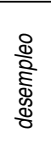 & 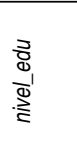 & $\begin{array}{l}\underset{\Phi}{\Xi} \\
\stackrel{\Phi}{\leftrightarrows}\end{array}$ & 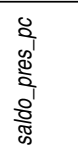 & $\begin{array}{l}0 \\
0 \\
\frac{8}{8} \\
\frac{8}{0}\end{array}$ & 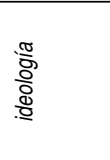 & 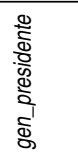 & 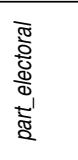 & 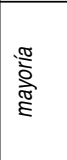 \\
\hline & 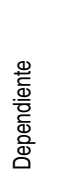 & \multicolumn{7}{|c|}{ 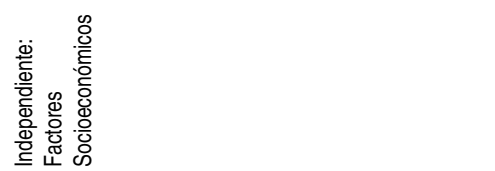 } & \multicolumn{4}{|l|}{ 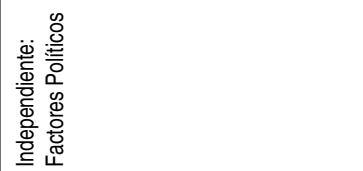 } \\
\hline
\end{tabular}




\subsection{Modelo}

Con el fin de analizar los factores explicativos de los niveles de transparencia autonómica presupuestaria, se plantea el siguiente modelo de regresión múltiple lineal:

trans_presi $=\alpha i+\beta 1 i^{*}$ poblacióni $+\beta 2 i$ * desempleoi $+\beta 3 i{ }^{*}$ nivel_edui $+\beta 4 i$ * internet $i+\beta 5 i$ * saldo_pres_pci $+\beta 6 i{ }^{*}$ deuda_pci $+\beta 7 i$ * ideología $+\beta 8 i^{*}$ gen_presidentei $+\beta 9 i{ }^{*}$ part_electorali + $\beta 10 i$ * mayoríai $+\varepsilon i$

donde el subíndice $i(i=1 \ldots 17)$ recoge las distintas CCAA, a es la constante de la ecuación, $\beta_{1} \ldots$ $\beta_{10}$ son los coeficientes a estimar y $\varepsilon$ es el término de error.

Este modelo se estimará mediante el método de los Mínimos Cuadrado Ordinarios (MCO), procedimiento que plantea utilizar como estimación de los parámetros aquella combinación de $\beta 1, \beta 2, \ldots$, $\beta \mathrm{j}$ que minimice los errores del modelo realizado.

Bajo los supuestos clásicos del modelo de regresión linear, los coeficientes resultantes de estimar mediante el método MCO son considerados lineales, insesgados, eficientes y consistentes. Sin embargo, si se regresa un modelo a través de MCO con presencia de heterocedasticidad, los coeficientes siguen siendo lineales, insesgados y consistentes, pero dejan de ser eficientes (mínima varianza) (Wooldridge, 2003).

En este sentido, aplicaremos el test de White para estudiar la presencia de heterocedasticidad en los modelos considerados. Este test contrasta la hipótesis nula de ausencia de heterocedasticidad sin suponer una forma funcional concreta para la varianza. En la tabla 5 se observa que en nuestro modelo no podemos rechazar la hipótesis nula a un nivel de significación del 1\%, por lo que consideramos que la varianza es constante y homocedástica. Por tanto, los coeficientes estimados mediante MCO tienen buenas propiedades estadísticas.

Por otra parte, hay que tener en cuenta que en presencia de multicolinealidad los estimadores están calculados de manera imprecisa. Esto se puede considerar un problema cuando la correlación entre las variables independientes es tan alta que se hace casi imposible estimar con precisión los efectos individuales de cada una de ellas. Así, se han realizado distintas pruebas para comprobar que nuestro modelo no adolece de multicolinealidad. En un principio, medimos el nivel económico de las Comunidades Autónomas utilizando la variable nivel_eco, pero como podemos ver en la tabla 3, esta variable presenta una alta correlación con las variables desempleo y nivel_edu, por lo que al final decidimos, tal y como ya hemos comentado en el apartado anterior, medir el nivel económico utilizando la variable desempleo. Una vez eliminada esta variable del modelo, ninguna de las variables independientes incluidas finalmente están muy correlaciones entre ellas (no observamos correlaciones por encima del 0,7 ), por lo que no hay multicolinealidad y el modelo tendrá buenas propiedades estadísticas. 


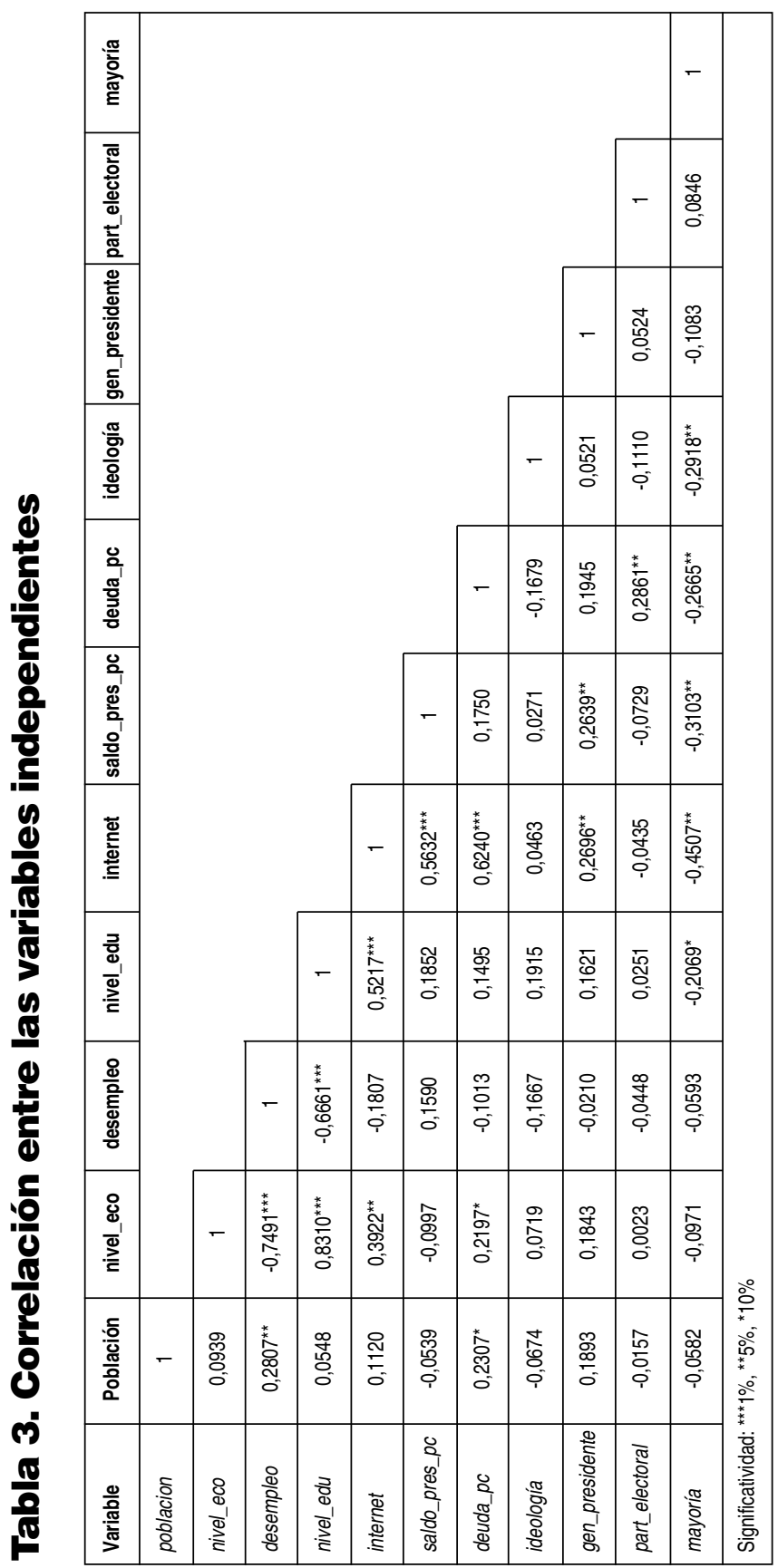


Además, comprobamos si existen problemas de multicolinealidad a través del cálculo del Factor de Inflación de la Varianza (FIV). Esta medida nos da el grado en que cada una de las variables independientes se explica por otras variables independientes. Un valor del FIV superior a 5 indica que puede existir multicolinealidad.

\section{Tabla 4. Cálculo del Factor de Inflación de la Varianza del modelo}

\begin{tabular}{|c|c|c|}
\hline Variable & FIV & 1/FIV \\
\hline internet............. & 4,59 & 0,22 \\
\hline nivel_edu & 3,82 & 0,26 \\
\hline desempleo .............. & 3,29 & 0,30 \\
\hline deuda_pc.... & 3,00 & 0,33 \\
\hline saldo_pres_pc... & 1,97 & 0,51 \\
\hline población ............ & 1,60 & 0,62 \\
\hline mayoría.............. & 1,50 & 0,66 \\
\hline part_electoral............. & 1,31 & 0,76 \\
\hline ideología ....... & 1,26 & 0,80 \\
\hline 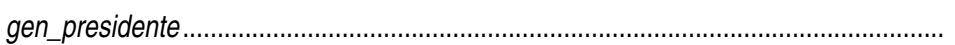 & 1,17 & 0,86 \\
\hline
\end{tabular}

Tal y como vemos en la tabla 4, ningún FIV es superior a 5, por lo que nuestro modelo final no presenta multicolinealidad. 


\section{Resultados}

La tabla 5 muestra los coeficientes obtenidos una vez estimado el modelo de regresión lineal para la variable explicativa del modelo (trans_pres) mediante MCO.

\section{Tabla 5. Regresión}

\begin{tabular}{|c|c|}
\hline VARIABLES INDEPENDIENTES & $\begin{array}{c}\text { VARIABLE DEPENDIENTE } \\
\text { trans_pres }\end{array}$ \\
\hline cte. & $38,04(0,83)$ \\
\hline población & $0,00(1,99)^{\star}$ \\
\hline desempleo & $-1,51(-2,31)^{\star \star}$ \\
\hline nivel_edu & $-0,95(-1,21)$ \\
\hline internet & $0,99(2,16)^{\star \star}$ \\
\hline saldo_pres_pc & $0,04(3,88)^{\star \star \star}$ \\
\hline deuda_pc & $-0,00(-0,14)$ \\
\hline ideología & $-2,48(-0.48)$ \\
\hline gen_presidente & $-14,74(-2,35)^{\star \star}$ \\
\hline part_electoral & $0,60(1,42)$ \\
\hline mayoría & $-3,81(-0,66)$ \\
\hline $\begin{array}{c}\mathrm{N}^{\circ} \text { Observaciones } \\
\mathrm{R}^{2} \\
\text { Test de White ( } \mathrm{p}(\text { chi-cuadrado)) }\end{array}$ & $\begin{array}{c}68 \\
0,55 \\
0,44\end{array}$ \\
\hline $\begin{array}{l}\text { Estimación Mediante MCO } \\
\text { t-valor ente paréntesis } \\
\text { Significatividad: }{ }^{* \star} 1 \%,{ }^{* \star} 5 \%,{ }^{*} 10 \%\end{array}$ & \\
\hline
\end{tabular}

En relación a la relevancia y al signo de cada una de las variables (ver tabla 5), podemos observar que la población afecta de manera muy leve al nivel de transparencia presupuestaria de las CCAA 
(trans_pres), pero lo hace de manera positiva y significativa. Esto significa que los gobiernos de las CCAA más pobladas tienen mayores niveles de transparencia presupuestaria con el objetivo de disminuir las asimetrías de información entre los gobernantes y los ciudadanos y permitir a estos últimos evaluar su actuación. Esto está en línea con lo encontrado en estudios anteriores, como el de Guillamón et al. (2011) para el caso de los municipios españoles.

Por otro lado, observamos como el desempleo afecta negativamente a los niveles de transparencia presupuestaria, es decir, las CCAA con unas mayores tasas de desempleo presentan, según el modelo, unos niveles de transparencia inferiores. Este resultado coincide con el de los estudios de Hammed (2005) y Piotrwski y Van Ryzin (2007), que encuentran que una mejor situación económica, como, por ejemplo, menores tasas de desempleo, favorece la transparencia presupuestaria.

Según los resultados obtenidos, no encontramos una relación significativa entre el nivel educativo (nivel_edu) y la transparencia presupuestaria (trans_pres), lo que coincide con las conclusiones de Serrano et al. (2009).

La variable internet sí que resulta estadísticamente significativa en el modelo, afectando positivamente a los niveles de transparencia, lo que prueba que las CCAA más transparentes son aquéllas en las que los ciudadanos tienen mayor acceso a internet. Este resultado está en línea con lo que argumenta Kim (2007), quien establece que cuando el número de ciudadanos con acceso a internet es elevado, la posibilidad de que éstos visiten las webs de sus gobiernos y demanden más información es mayor.

En cuanto al saldo_pres_pc, podemos observar que existe una relación positiva y significativa entre esta variable y la transparencia presupuestaria. Por tanto, nuestros resultados indican que aquellos gobiernos de las CCAA que tienen una mejor situación económica (mayor superávit) están dispuestos a divulgar una mayor cantidad de información presupuestaria, es decir, son más transparentes. Nuestro análisis empírico coincide con las conclusiones de Benito y Bastida (2009), que encuentran una correlación positiva y significativa entre el resultado presupuestario y la transparencia presupuestaria.

Finalmente, la variable deuda_pc no tiene un impacto significativo sobre la transparencia presupuestaria, lo que sugiere que el nivel de endeudamiento no afecta a la cantidad de información presupuestaria que divulgan los gobiernos autonómicos. Esto concuerda con Guillamon et al. (2011), que no consiguen demostrar que exista tal relación para el caso de los gobiernos locales españoles.

Respecto a las variables políticas, no encontramos una relación significativa entre la ideología y la transparencia presupuestara, coincidiendo con lo que concluyen Bastida y Benito (2007), Serrano et al. (2009) y Navarro et al. (2016) en la literatura previa. Esto significa que tanto los gobiernos progresistas como los conservadores de las distintas CCAA españolas tienen niveles similares de transparencia presupuestaria. 
En cuanto a la variable gen_presidente, nuestros resultados muestran una relación estadísticamente significativa entre el género del presidente autonómico y los niveles de transparencia presupuestaria. De hecho, nuestra investigación sugiere que los gobiernos autonómicos españoles gobernados por hombres son más transparentes que los gobernados por mujeres. En esta línea, Piotrowski y Van Ryzin (2007), siguiendo lo expuesto por Jennings (1983), sostienen que los hombres son más propensos a exigir una mayor transparencia, ya que éstos están más comprometidos con la política.

En el caso de la variable part_electoral, nuestro modelo no muestra una relación significativa entre la misma y los niveles de transparencia presupuestaria de las CCAA. Estos resultados coinciden con los obtenidos por estudios anteriores como los de La Porte et al. (2002).

Por último, el coeficiente de la variable mayoría tampoco es significativo, lo que revela que tanto los gobiernos autonómicos que gobiernan en mayoría como los que lo hacen en coalición presentan niveles similares de transparencia presupuestaria. En este caso, nuestro estudio está en la misma línea que las conclusiones de Gallego et al. (2010) y Cuadrado (2014), quiénes no encuentran relación entre la competencia política y la transparencia.

La tabla 6 resume las hipótesis inicialmente planteadas (ver sección 2) y si finalmente han sido aceptadas o rechazas tras el contraste de las mismas. 


\section{Tabla 6. Resumen de las hipótesis contrastadas}

\begin{tabular}{|l|l|}
\hline HIPÓTESIS & ACEPTADA/RECHAZADA \\
\hline $\begin{array}{l}\text { H1: Existe una relación positiva entre el tamaño de la población de las CCAA y la trans- } \\
\text { parencia presupuestaria de sus gobiernos. }\end{array}$ & ACEPTADA \\
\hline $\begin{array}{l}\text { H2: Existe una relación entre el nivel económico de las CCAA y la transparencia presu- } \\
\text { puestaria de sus gobiernos. }\end{array}$ & ACEPTADA \\
\hline $\begin{array}{l}\text { H3: Existe una relación positiva entre el nivel educativo de la población de las CCAA y la } \\
\text { transparencia presupuestaria de sus gobiernos. }\end{array}$ & RECHAZADA \\
\hline $\begin{array}{l}\text { H4: Existe una relación positiva entre el acceso a internet de la población de las CCAA y } \\
\text { la transparencia presupuestaria de sus gobiernos. }\end{array}$ & ACEPTADA \\
\hline $\begin{array}{l}\text { H5: Existe una relación entre el saldo presupuestario de las CCAA y la transparencia pre- } \\
\text { supuestaria de sus gobiernos. }\end{array}$ & ACEPTADA \\
\hline $\begin{array}{l}\text { H6: Existe una relación entre el nivel de endeudamiento de las CCAA y la transparencia } \\
\text { presupuestaria de sus gobiernos. }\end{array}$ & RECHAZADA \\
\hline $\begin{array}{l}\text { H7: Existe una relación entre la ideología del presidente de la CCAA y la transparencia pre- } \\
\text { supuestaria. }\end{array}$ & ACEPTADA \\
\hline $\begin{array}{l}\text { H8: Existe una relación entre el género del presidente de la CCAA y la transparencia pre- } \\
\text { supuestaria. }\end{array}$ & RECHAZADA \\
\hline $\begin{array}{l}\text { H9: Existe una relación positiva entre la participación de los ciudadanos en las eleccio- } \\
\text { nes autonómicas y la transparencia presupuestaria. }\end{array}$ & RECHAZADA \\
\hline $\begin{array}{l}\text { H10: Existe una relación entre el mayor grado de competencia política y la transparencia } \\
\text { presupuestaria. }\end{array}$ & RECHAZADA \\
\hline
\end{tabular}

\section{Conclusiones}

El objetivo principal de nuestro trabajo ha sido analizar cómo afectan diferentes determinantes a los niveles de la transparencia presupuestaria de los gobiernos autonómicos, utilizando como medida de ésta el índice de transparencia económico-financiera creado por Transparencia Internacional España. En concreto, hemos estudiado diversos factores socioeconómicos y políticos que pueden condicionar los niveles de transparencia presupuestaria por parte de los gobiernos autonómicos españoles. 
Este estudio se ha llevado a cabo utilizando una muestra constituida por todas las CCAA españolas para los años 2010, 2012, 2014 y 2016, siendo éstos los únicos para los que Transparencia Internacional ha elaborado este índice de transparencia económico-financiera.

Respecto a los factores socioeconómicos, nuestros datos muestran que el tamaño de la población, el uso de internet por parte de los ciudadanos y la capacidad de financiación de los gobiernos autonómicos tienen un impacto positivo sobre los niveles de transparencia presupuestaria de las CCAA. Por otro lado, la tasa de desempleo tiene un impacto negativo, es decir, cuanto mayor es ésta (peor situación económica), menor es la transparencia presupuestaria de los gobiernos autonómicos.

Asimismo, observamos que variables socioeconómicas como el nivel educativo de la población y el nivel de deuda de la CA no resultan relevantes para determinar los niveles de transparencia presupuestaria.

En cuanto a los factores políticos, encontramos que solo el género del presidente autonómico parece ser relevante respecto a los niveles de transparencia. En concreto, concluimos que las autonomías españolas gobernadas por hombres presentan un mayor nivel de transparencia presupuestaria que aquellas gobernadas por mujeres. No obstante, hay que tener en cuenta que los resultados obtenidos para esta variable pueden estar sesgados debido a que en nuestra muestra el número de presidentes autonómicos es bastante más elevado que el de presidentas. Consideramos, por tanto, esto como una limitación y creemos necesario la realización de estudios en el futuro que intenten arrojar más luz sobre la relación entre el género y la transparencia, un tema que sin duda está muy en boga hoy en día y del que queda mucho por investigar.

Otros factores políticos como la ideología, el grado de participación electoral o la forma de gobierno (mayorías o coaliciones) no son significativos en el estudio.

El análisis de la evolución del índice de transparencia económico-financiero de las CCAA nos ha permitido observar que su propia publicación ha sido uno de los mayores estímulos para que los gobiernos incrementen su nivel de información pública. Eso es un hecho que supone sin duda una limitación a la variable dependiente utilizada en nuestro modelo, ya que el rango de variación de ésta se va reduciendo en el tiempo, acercándose ya muchas CCAA a índices de transparencia del 100\% en 2016. Por tanto, es posible que, con el paso del tiempo y la publicación de nuevos índices de transparencia, el modelo propuesto en este estudio deje de ser significativo ya que la variable dependiente (el índice de transparencia) tenderá a ser uniforme (el valor del índice de todas las CCAA será o llegará a ser el $100 \%$ ). Además, no hay que olvidar que otra de las limitaciones propias de estos indicadores es que no incluyen en su análisis la calidad de la información emitida por los gobiernos, sino solamente la propia publicación de esta información.

Cabe destacar también que toda divulgación acarrea una serie de costes, fundamentalmente en tiempo de personal dedicado a la preparación de la información. No obstante, gran parte de la infor- 
mación se elabora periódicamente para el cumplimiento de obligaciones con otras instancias administrativas. Por ello, consideramos que el avance de la transparencia en el ámbito de las autonomías es una cuestión de predisposición, voluntad y saber hacer, más que de grandes inversiones tecnológicas.

En este sentido, creemos que los gobiernos autonómicos tendrían que optar por seguir mejorando sus niveles de transparencia, sobre todo aquellas autonomías de menor tamaño, ya que como hemos podido concluir en el estudio son las que tienen menores niveles de transparencia. Esto lo pueden conseguir a través de mejoras en los medios con los que comunican la información. Así, como hemos concluido, el acceso a internet de la población es un factor que afecta muy positivamente a los niveles de transparencia. Por lo tanto, las autonomías tienen que poner énfasis en este tipo de medios para proporcionar información de calidad y con suficiente claridad a los ciudadanos.

Normalmente, las instituciones tienden a hacer los datos públicos, pero no comprensibles para la gran parte de la ciudadanía. Por este motivo, el papel de los ciudadanos es seguir exigiendo a las administraciones públicas una información exhaustiva, clara y completa de su gestión.

Como líneas futuras de investigación que permitan complementar y profundizar el estudio de los niveles de transparencia sería positivo la creación de nuevos índices para poder comprobar las similitudes y diferencias entre éstos, incluyendo en el cálculo de estos índices la calidad y la claridad de la información que se publica por parte de las administraciones. También sería positivo analizar la evolución del índice de transparencia a través de datos de panel cuando exista un mayor espacio temporal.

\section{Bibliografía}

ALBALATE, D. (2013): "The institutional, economic and social determinants of local government transparency", Journal of Economic Policy Reform, 16(1), 90-107. DOI: 10.1080/17487870.2012.759422.

ALESINA, A. \& PEROTTI, R. (1996): "Fiscal Discipline and the Budget Process", American Economic Review, 86(2), 401-407.

ALONSO, M.L. \& GARCÍA, J. (2014): "Evaluación de la transparencia municipal en el Principado de Asturias", Auditoría Pública, 64, 75-86.

ALT, J.E., LASSEN, D. \& ROSE, S. (2006): "The Causes of Fiscal Transparency: Evidence from the U.S. States. IMF Staff Papers, 53, Special Issue, 30-57. 
ARIKAN, G. (2004): "Fiscal Decentralization: A remedy for Corruption?", International Tax and Public Finance, 11(2), 175-195. DOI: 10.1023/B:ITAX.0000011399.00053.a1.

BASTIDA, F. \& BENITO, B. (2007): "Central government budget practices and transparency: an international comparison", Public Administration, 85(3), 667-716.

DOI: 10.1111/j.1467-9299.2007.00664.x.

BENITO, B. \& BASTIDA, F. (2009): "Budget Transparency, Fiscal Performance, and Political Turnout: An International Approach", Public Administration Review, 69(3), 403-416. DOI: 10.1111/j.15406210.2009.01988.x.

BELLOD, J. (2008): "Significado y evaluación de la transparencia presupuestaria en las Comunidades Autónomas", Auditoría Pública, 45, 103-115.

CHRISTIAENS, J. (1999): "Financial accounting reform in Flemish municipalities: An empirical investigation", Financial Accountability and Management, 15(1), 21-40. DOI: 10.1111/1468-0408.00072.

CUADRADO-BALLESTEROS, B. (2014): "The impact of functional decentralization and externalization on local government transparency", Government Information Quarterly, 31 (2), 265-277. DOI: 10.1016/j.giq.2013.10.012.

ESTELLER-MORÉ A. \& POLO-OTERO, J. (2012): "Fiscal Transparency: (Why) does your local government respond?", Public Management Review, 14(8), 1153-1173.

DOI: 10.1080/14719037.2012.657839.

EVANS, J.H. \& PATTON, J.M. (1987): "Signaling and monitoring in public-sector accounting", Journal of Accounting Research, 25, 130-158.

FEREJOHN, J. (1999): Accountability and authority: towards a model of political accountability. En A. Przeworski, S.C. Stokes and B. Manin (Eds), Democracy, Accountability and Representation, New York: Cambridge University Press, 131-153.

FONDO MONETARIO INTERNACIONAL (2012): Transparencia Fiscal, rendición de cuentas y riesgo fiscal, Washington: International Monetary Fund.

GALLEGO-ÁLVAREZ, I., RODRÍGUEZ-DOMÍNGUEZ, L. \& GARCÍA-SÁNCHEZ, I.M. (2010): "Are determining factors of municipal E-government common to a worldwide municipal view? An intracountry comparison", Government Information Quarterly, 27(4), 423-430. DOI: 10.1016/j.giq.2009.12.011.

GANDÍA, J.L. \& ARCHIDONA, M.C. (2008): "Determinants of Web site information by Spanish city councils", Online Information Review, 32(1), 35-57. DOI: 10.1108/14684520810865976.

GARCíA, J. (2014): "Gobierno abierto: transparencia, participación y colaboración en las administraciones públicas", Innovar, Revista de Ciencias Administrativas y Sociales, 24(54), 75-88. DOI: 10.15446/innovar.v24n54.46441. 
GARCÍA-TABUYO, M., SÁEZ-MARTIN, A. \& CABA-PÉREZ, M. (2015): "Proactive Transparency Policy in the Mercosur Local Governments: Regulation vs. Self-Regulation", Transylvanian Review of Administrative Sciences, 11(46), 71-90.

GIROUX, G. \& MCLELLAND, A. (2003): "Governance structures and accounting at large municipalities", Journal of Accounting and Public Policy, 22(3), 203-230. DOI: 10.1016/S0278-4254(03)00020-6.

GRIGORESCU, A. (2003): "International Organizations and Government Transparency: Linking the International and Domestic Realms", International Studies Quarterly, 47(4), 643-667. DOI: 10.1046/j.0020-8833.2003.04704003.x.

GUILLAMÓN, M.D., BASTIDA, F. \& BENITO, B. (2011): "The determinants of Local Government's Financial transparency", Local Government Studies, 37(4), 391-406.

DOI: 10.1080/03003930.2011.588704.

HAMMED, F. (2005): "Fiscal Transparency and economic outcomes", IMF Working Paper, Washington: International Monetary Fund.

HUTHER, J. \& SHAH, A. (1998): "A Simple Measure of Good Governance and its Application to the Debate on the Appropriate Level of Fiscal Decentralization", World Bank Policy Research Working Paper Series 1894, Washington, DC: Work Bank.

INGRAM, R.W. \& DEJONG, D.V. (1987): "The effect of regulation on local government disclosure practices", Journal of Accounting and Public Policy, 6(4), 245-270. DOI: 10.1016/S0278-4254(87)800029.

JUNQUERA, E.L. (2013): "Economía y transparencia en un contexto mundial", Encuentros Multidisciplinares, 43, 63-71.

KIM, C.K. (2007): "A cross-national analysis of global E-government", Public Organization Review, 7(4), 317-329. DOI: 10.1007/s11115-007-0040-5.

KOPITS, G. \& CRAIG, J. (1998): Transparency in Government Operations, Washington, DC: Work Bank.

LA PORTE, T.M., DEMCHAK, C.C. \& JONG, M. (2002): "Democracy and Bureaucracy in the Age of the Web: Empirical Findings and Theoretical Speculations", Administration and Society, 34(4), 411446. DOI: $10.1177 / 0095399702034004004$.

MA, L. \& WU, J. (2011): "What Drives Fiscal Transparency? Evidence from Provincial Government in China", 1st Global Conference on Transparency Research, Rutgers University-Newark.

MITCHELL, R.B. (1998): "Sources of Transparency. Information Systems in International Regimes", International Studies Quarterly, 42(1), 109-130. DOI: 10.1111/0020-8833.00071.

NAVARRO, E., MORA, M. \& DELGADO, M.L. (2016): "Analyzing the transparency traditional variables within the Spanish municipalities", Transylvanian Review of Administrative Sciences, 12(47), 129145. 
PALLOT, J. (2001): "Transparency in local government: antipodean initiatives", European Accounting Review, 10(3), 645-660. DOI: 10.1080/09638180126794.

PINA, V., TORRES, L. \& ROYO, S. (2007): "Are ICTs improving transparency and accountability in the EU regional and local governments? An empirical study", Public Administration, 85(2), 449-472. DOI: 10.1111/j.1467-9299.2007.00654.x.

PIOTROWSKI, S.J. \& VAN RYZIN, G.G. (2007): "Citizen attitudes toward transparency in local government", The American Review of Public Administration, 37(3), 306-323.

DOI: $10.1177 / 0275074006296777$.

POLO-OTERO, J. (2011): "Análisis de los determinantes de la transparencia fiscal: Evidencia empírica para los municipios catalanes", Revista de Economía del Caribe, 8, 133-166.

RíOS, A., BASTIDA, F. \& BENITO, B. (2013): "Determinants of Central Government Budget Disclosure: An International Comparative Analysis", Journal of Comparative Policy Analysis: Research and Practice, 15(3), 235-254. DOI: 10.1080/13876988.2013.798449.

RÍOS, A., BASTIDA, F. \& BENITO B. (2014): "Budget Transparency and Legislative Budgetary Oversight: An International Approach", The American Review of Public Administration, 46(5), 546568. DOI: $10.1177 / 0275074014565020$.

SERRANO-CINCA, C., RUEDA-TOMÁS, M. \& PORTILLO-TARRAGONA, P. (2009): "Factors influencing e-disclosure in local public administrations", Environment and planning C: Government and Policy, 27(2), 355-378. DOI: 10.1068/c07116r.

TRANSPARENCIA INTERNACIONAL ESPAÑA (2018): Transparencia Internacional España [sitio web]. Madrid: Transparencia Internacional España [Consulta: 20 de febrero de 2018]. Disponible en: https: //transparencia.org.es/indice-de-las-comunidades-autonomas-incau/

TOLBERT, C.J. \& MOSSBERGER, K. (2006): "The effects of e-government on trust and confidence in government", Public Administration Review, 66(3), 354-369.

DOI: 10.1111/j.1540-6210.2006.00594.x.

VeRBA, S., NIE, N.H. \& KIM, J. (1978): Participation and Political Equality: A Seven-Nation Comparison, New York: Cambridge University Press.

WILSON, E. \& HOWARD, T. (1985): "Information for municipal bond investment decisions: synthesis of prior research, and extension and policy implications", Research in Governmental and Nonprofit Accounting, 1, 213-263.

WOOLDRIDGE, J.M. (2003): Introductory Econometrics: $A$ Modern Approach, $2^{\circ}$ ed. Boston: Southwestern College Publishing.

ZIMMERMAN, J.L. (1977): "The municipal accounting maze: an analysis of political incentives", Journal of Accounting Research, 15, 107-44, supplement. 